\title{
LOW-COHERENCE INTERFEROMETRY FOR MEASUREMENT OF PROPERTIES OF OPTICAL COMPONENTS
}

We present a modification of the interferometric method for the measurement of refractive index or thickness of optical devices using an easily aligned, almost all-fiber Michelson interferometer. Applicability of the method is demonstrated by the chromatic dispersion measurement of photonic crystal fiber sample. The birefringence of the optical device based on $\mathrm{LiNbO}_{3}$ obtained from the measurement of refractive indices is demonstrated and its value is determined for two different crystal samples. It is also shown that when the thickness of, for example, photopolymer material is known the refractive index of the device can be determined. The described method can be a practical tool for laboratories with the need of inexpensive and easily built setup for measurement of refractive index or thickness of optical devices even in a broad spectral range.

\section{Introduction}

Generally, the techniques of optical interferometry play a crucial role among the measurement techniques used in optics. Nowadays, many of the techniques employ optical fibers as function part of the interferometer and both, the time-domain and the spectral-domain optical interferometric techniques can be applied to measure various parameters of optical fibers and devices [1,2]. One of the possibilities of investigation of optical devices' parameters is low coherence interferometry [3]. The appropriate interferometer can be built using bulk optical components [4] or as all (or almost all) fiber interferometer [5, 6]. The principle of methods lies in balancing the optical lengths of two arms of interferometer, where the measured sample is placed in the test arm and the reference arm contains the variable optical delay line.

In the paper the authors discuss the utilisation of the low coherence interferometry for the determination of useful parameters of simple devices for integrated optics such as wave plates and thin photopolymer substrate layers for optical memories. The applicability of the method is demonstrated on the measurement of chromatic dispersion of a sample of photonic crystal fiber.

Photonic crystal fibers [7] offer new optical properties comparing to conventional optical fibers. Especially their dispersion characteristics open up the space for new applications (e.g. supercontinuum generation even in the visible spectrum). Due to the fact that photonic crystal fibers may exhibit large longitudinal inhomogeneity or have large attenuation the interferometric method of chromatic dispersion measurement is often preferred. The dispersion characteristics can be obtained by measuring the group delays $\tau_{g}$ from the interferograms' envelope for several discrete wavelengths $\lambda_{i}$. The measured data $\tau_{g}\left(\lambda_{i}\right)$ are fitted by an analytical function, e.g. by three- or five- term Sellmeier function. The dispersion coeficient is then given by the relation $D(\lambda)=1 / L d \tau_{g} / d \lambda$, where $L$ is the length of the investigated fiber.

Photosensitive crystal lithium niobate $\left(\mathrm{LiNbO}_{3}\right)$ is one of the best and widely used materials for integrated optics due to its excellent electrooptical and photorefractive properties. Most often the thin plates of the bulk material are used either as simple retarders (wave plates) or as substrates for elements of integrated optics such as waveguides, gratings, switches, directional couplers, optical modulators etc. The bulk crystal is also often regarded as a promissing medium for holographic memory. Except the crystals there are the various kinds of photopolymers the photosensitive properties and cost of which started competing with the crystalline materials during last decade. Nevertheless, the important physical parameters of optical devices whether made of crystalline or photopolymer materials are the refractive index and their thickness. Both these parameters can be determined by low coherence interferometry.

\section{Theory}

The intensity $I$ of interference pattern of two polychromatic beams with angular frequency falling into interval $\omega_{0} \pm \Delta \omega / 2$ can be expressed as [8]

$$
I=I_{1}+I_{2}+2 \gamma_{1,2} \sqrt{I}_{1} I_{1} \cos (\varphi),
$$

\footnotetext{
* Daniel Kacik, Norbert Tarjanyi, Ivan Turek

Department of Physics, Faculty of Electrical Engineering, University of Zilina, Slovakia, E mail: kacik@fel.uniza.sk
} 
where $I_{1}$ and $I_{2}$ are intensities of interfering beams, $\phi=\omega \tau$ is the phase difference between the interfering beams, and $\gamma_{12}$ is the interference visibility (or "interference contrast").

If the spectral density of ligth intensity is constant in the interval $\omega_{0} \pm \Delta \omega / 2$, then

$$
\gamma_{1,2}=\frac{\sin \left(\Delta \omega \tau_{g}\right)}{\Delta \omega \tau}
$$

where $\tau=\frac{\left(n_{1} l_{1}-n_{2} l_{2}\right)}{c}$ is the time delay difference between the beams $\left(n_{1,2}\right.$ and $l_{1,2}$ means refractive indices and lengths of the arms of the interferometer, respectively).

One arm of the interferometer contains

$$
\begin{aligned}
& n_{1} l_{1}=n_{\text {eff }}^{S M F} \times L^{\text {ref }}+L^{\text {air }}+n_{\text {eff }}^{\text {objejtive }} \times L^{\text {objective }}+ \\
& +n_{\text {eff }}^{\text {sample }} \times L^{\text {sample }}
\end{aligned}
$$

and second arm

$$
n_{2} l_{2}=n_{e f f}^{S M F} \times L^{\text {test }}+n_{\text {eff }}^{F U T} \times L^{F U T}
$$

where $n_{\text {eff }}$ is the effective refractive index of the particular media, $L^{r e f}$ and $L^{\text {test }}$ are the fiber lengths of the coupler branch in the reference and test arm, respectively. The difference of fiber lengths of the coupler was less than $0.3 \% . L^{F U T}, L^{\text {air }}$ and $L^{\text {sample }}$ are the lengths of the optical fiber, the air path and thickness of optical devices, respectively. $L^{\text {objective }}$ is the length of the microscope objective. In our preliminary work [9] we showed the influence of the microscopic objective on determination of chromatic dispersion of a conventional telecommunication fiber.

The interference term (the third term in right hand side of Eq.(1)) has a maximum when $n_{1} l_{1}=n_{2} l_{2}$ which allows to determine the refractive index of one arm if the lengths of the arms and refractive index of the second arm are known.

\section{Experiment}

Schematic layout of the experimental setup is shown in Fig.1 The beam splitter of the Michelson interferometer is made of a fiber coupler with 50:50 coupling ratio at the wavelength of $1550 \mathrm{~nm}$. However, the coupling ratio is still suitable at the range of wavelengths from $1200 \mathrm{~nm}$ to $1700 \mathrm{~nm}$.

A halogen lamp and Carl Zeiss monochromator or broadband optical fiber light source OFLS B-15-75-C-F-A was used as the wideband, low coherent light source. The reference arm of the interferometer is composed of a variable-optical-delay line and microscope objective (Meopta $6 \mathrm{x}, \mathrm{NA}=0.15$ ) to project the end of the fiber onto a mirror. The advantage of this setup is that eventual imperfections of the used mirror are minimized. Additionally, the focusing of the beam is easier than its colimation. The reflected

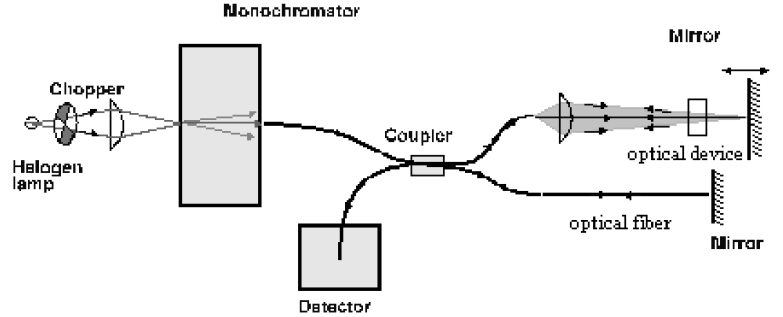

Fig. 1 The experimental setup of the interferometer

beam can be aimed right into the core of the fiber by proper orientation of the mirror. Moreover, the samples (optical devices) under investigation are put into the path of the ligth beam in the air line arm. The length of the variable-optical-delay line is changed using coarse translation in a range of $20-150 \mathrm{~cm}$ and fine translation controlled by a micrometric screw in the range of $25 \mathrm{~mm}$. This allows us to find very precisely the position of maximal interference visibility. The microscope objective is also located on a translation stage with micrometric sensitivity - this enables us to correct the spectral dependence of the focal length of the lens. A change of its location is needed for projecting the fiber core back to the fiber core so it does not influence the optical path in the variableoptical-delay line like it is in the case when the mirror is moving. Another way to make the variable optical delay line is to put the fiber into reference arm and balance the optical lengths of two arms interferometer by the change of fiber length [5]. Thus the interferometer can be made all-fiber and alignment-free.

The fiber under test is usually connected to the fiber coupler using bare-fiber adapters. In case of characterisation of fibers with non-standard diameter or with highly off-set core, the fibers have to be aligned using 3D micro-stage. The reflection at the end-face of the measured fiber is usually obtained by a chemical deposition of silver at the cleaved end-face [10]. When investigating the PCF a mirror cannot be created at the end of a fiber because of the air holes in structure of waveguide. For this reason we put PCF into a connector and placed it perpendicular to the mirror using the jig for polishing of the fiber end-face [3]. A disadvantage of the arrangement is a risk of damage of the mirror and fiber end-face.

Interference of the signal from the output end of the fiber coupler was detected by a detector with $\mathrm{GaAs}$ or $\mathrm{Si}$ photodiode and recorded by a phase sensitive amplifier and an oscilloscope, respectively.

According to Eq. (2) the width of the interval of delay (i.e. the interval of optical path difference), in which the interference contrast significantly differs from zero, depends on the spectral width $\Delta \lambda$ of the beams and for $\Delta \lambda=20 \mathrm{~nm}$ it is about $0.08 \mathrm{~mm}$ as illustrated in Fig. 2.

The measured interferogram is the Fourier transform of an almost rectangular spectrum determined by a monochromator output slit aperture. 


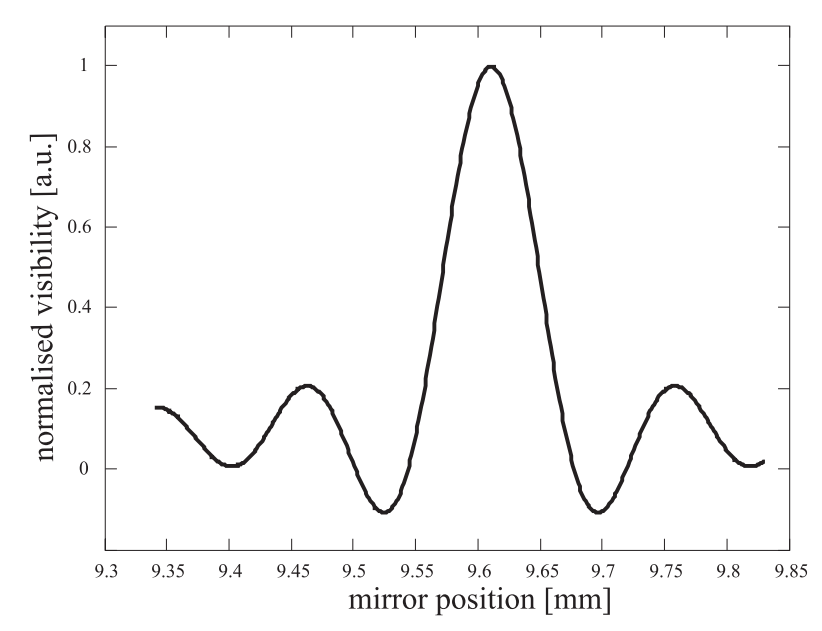

Fig. 2 The mirror position dependence of the interference term of conventional fiber measured at $1550 \mathrm{~nm}$ and $\Delta \lambda=20 \mathrm{~nm}$

The accuracy of determination of optical path difference between the arms (given by the accuracy of determination of the visibility maximum position) is better than $0.01 \mathrm{~mm}$ for the light with a spectral width of $20 \mathrm{~nm}$. The resolution of delay difference of interfering beams is of order of $0.01 \mathrm{ps}$.

\section{Results and Discussion}

We measured chromatic dispersion of PCF labeled as ASC014_B5a fabricated at OFTC (Optical Fibre Technology Centre) University of Sydney. This fiber is singlemode starting at $1000 \mathrm{~nm}$ and has a core diameter of $13 \mu \mathrm{m}$. An SEM image of a cleaved cross-section of its triangular lattice is shown in Fig. 3a.

The group delay, $\tau$, as a function of wavelength is measured and this is used to extract the fiber dispersion from the equation:

$$
D(\lambda)=\frac{1}{L} \frac{d \tau_{g}}{d \lambda}
$$

where $L$ is the length of measured fiber. The obtained dependencies are shown in Fig. $3 b$.

The analysis of the measurement of chromatic dispersion of PCF implies that using the experimental set up allows to determine the refractive index of transparent, about $1 \mathrm{~cm}$ thick samples with accuracy as precise as $10^{-4}$. Such precision allows, for example, to determine the birefringence of crystals, investigate the dependence of their refractive index value on impurities or when the refractive index is known then the thickness of the sample is possible to determine from this measurement. For investigation of the birefringence of crystals two samples of $\mathrm{LiNbO}_{3}$ with different amount of $\mathrm{Fe}$ ions and thicknesses were used. The optical axis of the crystal sample was perpendicular to the centre-line of the interferometer's test arm. Due to uniaxiality of the crystal and its alignment according to the centre-line of the interferometer's arm light incident on the crystal's front side is propagating in two polarization states inside the crystal. The ordinary and extraordinary waves travel different optical paths due to different values of refractive indices the waves experience while propagating throughout the crystal. The optical lengths of the test arm are different for ordinary and extraordinary polarized beams. It means that the interference patterns (visibility of interference) for ordinary and extraordinary polarizations will be separated from each other (Fig. 4). The separation of the maxima in the interference pattern is proportional to the birefringence of the crystal. For crystals measured we got values $\Delta n=0.081$ and $\Delta n=0.078$. Since the composition of the crystals was slightly different (different Fe doping level) the result implies the possibility to use the low-coherence interferometry for investigation of the dependence of the refractive index value of crystals on impurities.

A similar measurement can be done for any appropriate sample not necessarily a birefringent one. For the results presented here

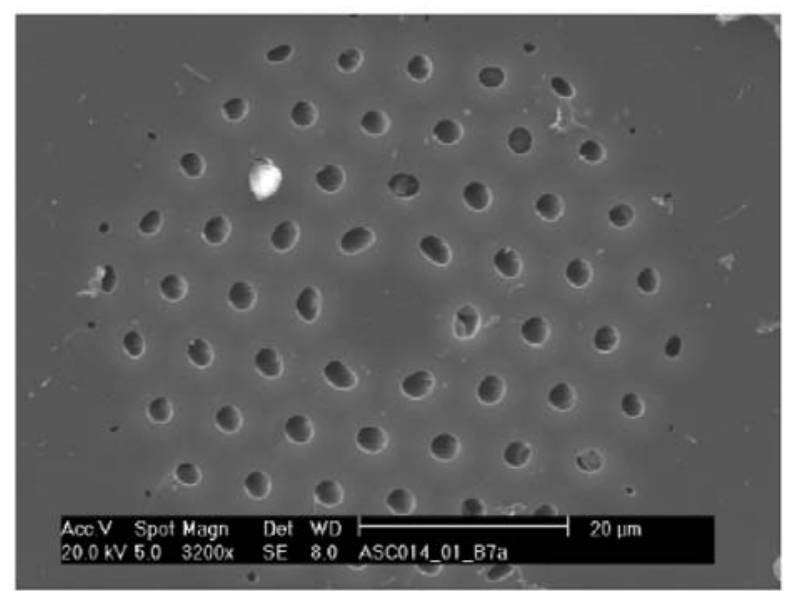

a)

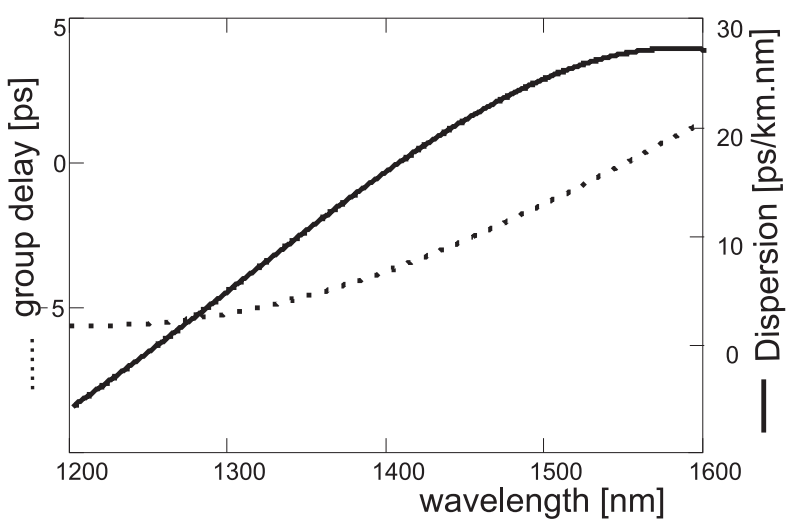

b)

Fig. 3 a) SEM image of cross section of photonic crystal fiber, b) spectral dependance of group delay and dispersion 


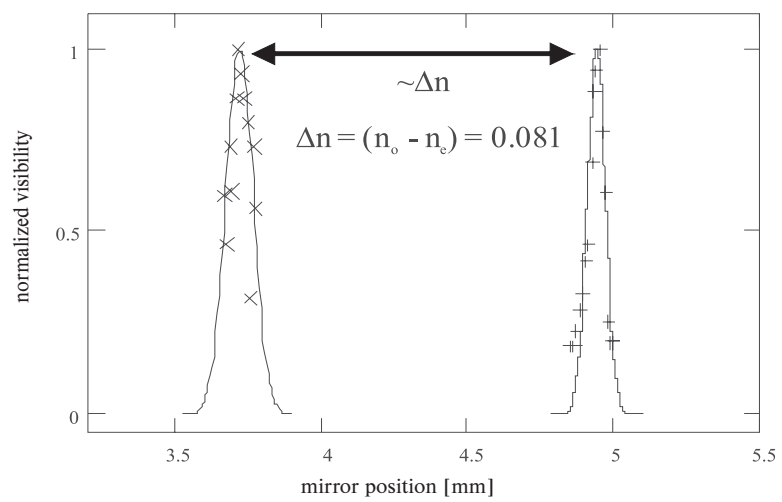

a)

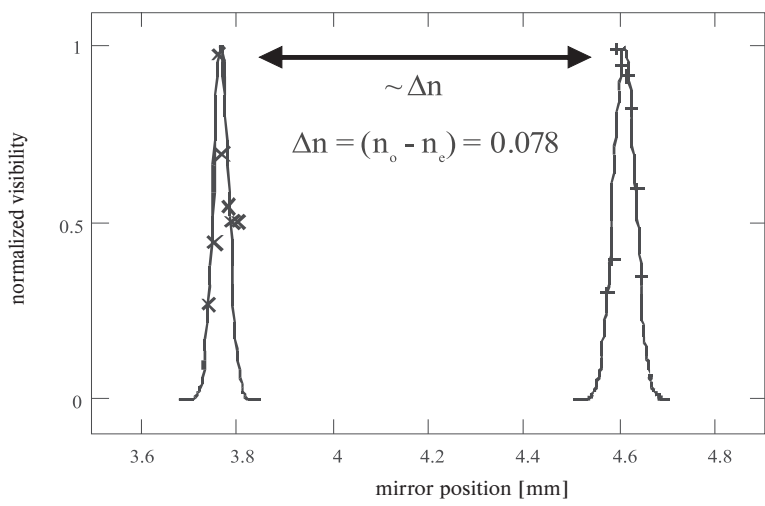

b)

Fig. 4 Birefringence of $\mathrm{LiNbO}_{3}$ with different content of Fe ions imaged by visibility of interference measured at $1550 \mathrm{~nm}$. The thickness of the samples was a) $15.055 \mathrm{~mm}$ and b) $10.765 \mathrm{~mm}$.

a polyvinyl-alcohol/acrylamide (PVA/AA) dry material was deposited on the glass plate. The material properties and its response to light are studied because of its intended utilization in holography [11]. The thicknesses of the sample layers were measured by means of a micrometer screw gauge and were about $l=135 \mu \mathrm{m}$ on average. The recent methods of investigation of the material properties require to know the values of the refractive indices of PVA/AA at some wavelengths laying in the visible part of light spectrum. However, for some applications one may need to know the refractive index value for a wavelength in a near infrared region. To estimate the refractive index of PVA/AA at $1310 \mathrm{~nm}$ we used a Safibra SLED OFSL-B-13-10-C-FA as a light source. First, a clean glass substrate without photopolymer layer was put into the test arm of the interferometer, the optical lengths of the interferometer's arms were balanced and the maximum visibility of interference fringes was measured. Then the glass substrate with a layer of the sample was put into the test arm and the optical lengths of the both arms were balanced again. The particular visibilities of inter-

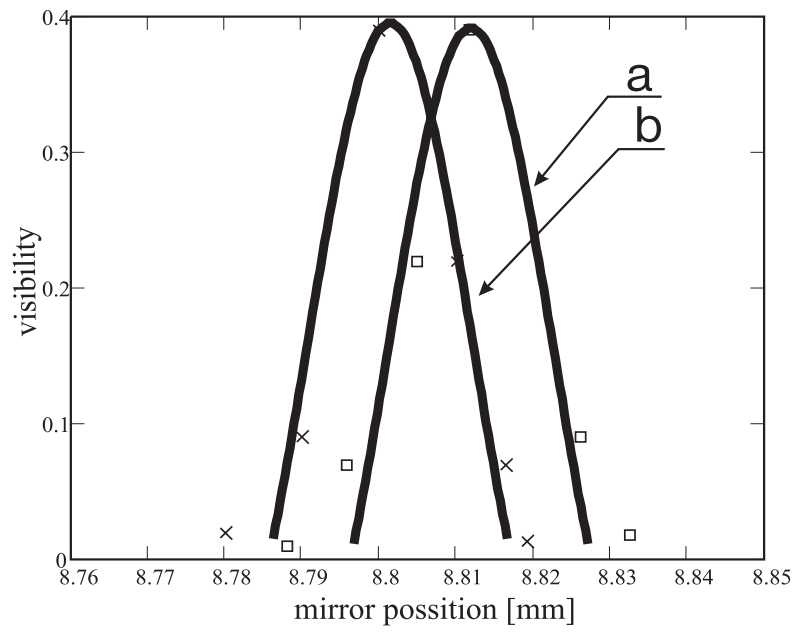

Fig. 5 Visibility function of interference for a) clean glass substrate, b) glass substrate with the photopolymer layer in the test arm of interferometer. Measured at $1310 \mathrm{~nm}$ ference are shown in Fig. 5. The distance between peaks of the curves shown in Fig. 5 is the change of the optical path due to a photopolymer layer. After readout of the displayed data we get a value of $0.015 \mathrm{~mm}$ for the optical path change and 1.11 for the refractive index of PVA/AA at $1310 \mathrm{~nm}$. Knowing the refractive index of PVA/AA at some wavelengths [e.g. 12] as well as the character of the spectral dependence of the refractive index according to Sellmeier equation for most of optical materials we can treat the resulting value as reasonable.

\section{Conclusion}

A method of refractive index measurement of optical devices using easily built optical-interferometer-based setup was presented. In contrast to other interferometric methods, it does not require special care for maintaining the interferometer in perfectly stable conditions and at the same time, it offers high accuracy of the group delay determination when investigating the fiber samples. The accuracy of the group delay measurement is $10 \mathrm{fs}$. The applicability of the presented method was demonstrated on measurement of chromatic dispersion of a sample of photonic crystal fiber. Using the setup we demonstrated the birefringence of the optical device based on $\mathrm{LiNbO}_{3}$ and from the measurement of refractive indices obtained the values of the birefringence for two different samples of iron doped $\mathrm{LiNbO}_{3}$. We also estimated the value of the refractive index of a sample of photopolymer material with the known thickness at the wavelength of $1310 \mathrm{~nm}$. The presented method can be a practical tool for laboratories where exists the need of inexpensive and easily built setup for measurement of refractive index or the thickness of optical devices even in a broad spectral range.

\section{Acknowledgements}

The authors wish to thank for the support to the R\&D operational program Centre of excellence of power electronics systems and materials for their components. The project is funded by the European Community, ERDF - European regional development fund. 


\section{COMMNICAIIONS}

\section{References}

[1] HLUBINA, P.: Measurement of Optical Fibers by Interferometric Methods. Proc. of SPIE (Optical Fibers: Applications, Warsaw 2005), 5952, p. 192-203, 2005.

[2] TUREK, I., TARJANYI, N.: Investigation of Symmetry of Photorefractive Effect in LiNbO 3 , Opt. Express (15)17, p. 10782-10788, 2007.

[3] PETERKA, P., KANKA, J., HONZATKO, P., KACIK, D.: Measurement of Chromatic Dispersion of Microstructure Optical Fibers Using Interferometric Method, Optica Applicata, 38(2): p. 295-303, 2008.

[4] HLUBINA, P.: White-light Spectral Interferometry to Measure the Effective Thickness of Optical Elements of Known Dispersion. Acta Physica Slovaca, 55 no. 4, p. 387-393, 2005.

[5] THEVENAZ, L. et al.: All-fiber Interferometer for Chromatic Dispersion Measurements, J. of Lightwave Technology, vol. 6, p. 1-7, 1988.

[6] KACIK, D., PETERKA, P., CANNING, J., TUREK, I., KOLIMAR, M., BEREZINA, S.: The Modified Interferometer for Measurement of the Chromatic Dispersion in PCFs, in Photonic Crystal Fibers, Proc. SPIE, Vol. 6588, 65880N, 2007.

[7] BJARKLEV, A., BROENG, J., BJARKLEV, A. S.: Photonic Crystal Fibres, Kluwer Academic Publishers, Boston, MA, 2003.

[8] KACIK, D., TUREK, I., TARJANYI, N.: Measurement of Modal Dispersion by Low Coherence Interferometer, Proc. SPIE, Vol. 7141, 71411K, 2008.

[9] BEREZINA, S., TUREK, I., KACIK, D.: Practical Arrangement of Interferometer for Optical Fiber Chromatic Dispersion Measurements, APCOM 2006, p. 312-317, 2006.

[10] DYMAK, P., PETERKA, P.: Chromatic Dispersion Measurement of Er doped Optical Fibers (in Czech), In sborník konference Opticke komunikace 2002, Praha, p. 136-140, 2002.

111] LAWRENCE, J. R., O’NEILL, F. T., SHERIDAN, J. T.: Photopolymer Holographic Recording Material, Optik 112(10), p. 449-463, 2001.

[12] GLEESON, M.R. et al.: Improvement of the Spatial Frequency Response of Photopolymer Materials by Modifying Polymer Chain Length, J. Opt. Soc. Am. B, 25(3), p. 396-406, 2008. 